\title{
Terapia ocupacional e oncologia pediátrica: caracterização dos profissionais em centros de referência no Estado de São Paulo
}

\section{Occupational therapy and pediatric oncology: characterization of professionals from reference health centers in the State of São Paulo}

\author{
Regina Helena Vitale Torkomian Joaquim ${ }^{1}$, Fernanda Brioschi Soares ${ }^{2}$, \\ Mirela de Oliveira Figueiredo ${ }^{3}$, Cristiane Miryam Drumond de Brito ${ }^{4}$
}

http://dx.doi.org/10.11606/issn.2238-6149.v28i1p36-45

Joaquim RHVT, Soares FB, Figueiredo MO, Brito CMD. Terapia ocupacional e oncologia pediátrica: caracterização dos profissionais em centros de referência no Estado de São Paulo. Rev Ter Ocup Univ São Paulo. 2017 jan.-abr.;28(1):36-45.

\begin{abstract}
RESUMO: O terapeuta ocupacional auxilia o paciente e a família a enfrentar e compreender o processo corporal e psíquico que a criança poderá passar a partir do momento que recebe o diagnóstico do câncer. No dia a dia visa melhorar a qualidade de vida da criança por meio de reabilitação, prevenção de sequelas, manutenção das atividades inerentes a faixa etária, além de proporcionar a (re) descoberta de capacidades e habilidades. O presente estudo teve por objetivo caracterizar os terapeutas ocupacionais que atuam na oncologia pediátrica do Estado de São Paulo. Trata-se de uma pesquisa descritiva, transversal e com abordagem quanti-qualitativa. A amostra se constituiu por 11 profissionais que atuam em instituições oncológicas. A coleta de dados ocorreu via internet, por questionário semiestruturado com questões fechadas e abertas sobre a formação e prática do profissional. Os profissionais participantes da pesquisa foram todos do sexo feminino, a faixa etária prevalente foi dos 25 aos 35 anos e a conclusão da graduação com maior incidência foi nos anos de 1980 e 2008. A abordagem terapêutica ocupacional predominante entre os profissionais teve como base o referencial da Terapia Ocupacional Dinâmica. As intervenções dos profissionais preconizam o trabalho multiprofissional, utilizam as brinquedotecas como local de intervenção e trabalham com uma diversidade de materiais em diferentes tipos de atividades. O quantitativo de terapeutas ocupacionais foi reduzido apesar de existirem 101 instituições que oferecem o tratamento oncológico. O tipo de atuação descrita pelos terapeutas ocupacionais denota responder aos preceitos da política de saúde no cuidado integral e ampliado.
\end{abstract}

DESCRITORES: Oncologia; Neoplasias; Criança; Terapia ocupacional.
Joaquim RHVT, Soares FB, Figueiredo MO, Brito CMD. Occupational therapy and pediatric oncology: characterization of professionals from reference health centers in the State of São Paulo. Rev Ter Ocup Univ São Paulo. 2017 Jan.-Apr.;28(1):36-45.

\begin{abstract}
Occupational therapists help patients and their families to confront and understand the body and psychic process that children may go through when they receive the diagnosis of cancer. In daily life, it aims to improve the quality of the life of children through rehabilitation, prevention of sequelae, maintenance of activities inherent to the age group, in addition to providing the (re)discovery of abilities and skills. This study aimed to characterize the occupational therapists who work with pediatric oncology in the state of São Paulo in Brazil. This is a cross-sectional descriptive research with quantitative-qualitative approach. The sample consisted of 11 professionals who work at oncology institutions. Data collection occurred via Internet, through a semi-structured questionnaire with closed and open questions about the professional's training and practice. The professionals who took part in the research were all female, with prevalent age range from 25 to 35 , and graduation conclusion with the highest prevalence was between 1980 and 2008. The predominant occupational therapy approach of these professionals was based on Dynamic Occupational Therapy. The interventions of the professionals preconize multi-professional work, use toy libraries as places of intervention and work with a number of different materials in different types of activities. The number of occupational therapists has been reduced, although there are 101 institutions offering oncological treatment. The type of action described by the occupational therapists responds to precepts of the health policy in integral and expanded care.
\end{abstract}

KEYWORDS: Medical oncology; Neoplasms; Child, Occupational therapy.

O presente trabalho constitui parte integrante de projeto de Iniciação Científica mediante financiamento do Programa Integrado de Apoio ao Docente recém-doutor pela Universidade Federal de São Carlos.

Parte dos dados desta pesquisa foram apresentados: I Congresso de Terapia Ocupacional em Contextos Hospitalares e Cuidados Paliativos, Ribeirão Preto, SP, nov. 2014; XIII Congresso Brasileiro de Terapia Ocupacional - Terapia Ocupacional e Políticas Públicas: diretrizes, compromissos e ações. Florianópolis, SC, out. 2013; XX Congresso de Iniciação Cientifica da UFSCar - Universidade Federal de São Carlos, jan. 2013; II Simpósio Internacional de Pesquisa em Terapia Ocupacional, São Paulo, SP, ago. 2012.

1. Docente do Departamento de Terapia Ocupacional, Programa de Pós-Graduação em Terapia Ocupacional e Programa de Pós-Graduação em Gestão da Clínica, Universidade Federal de São Carlos. E-mail: joaquimrhvt@gmail.com

2. Bacharel em Terapia Ocupacional pela Universidade Federal de São Carlos. Pós Graduada em Terapia Ocupacional aplicada à Neurologia pelo Hospital Israelita Albert Einstein. Residente Multiprofissional em Saúde Urgência e Trauma PUC Campinas. E-mail: fernandabris@yahoo.com.br 3.Docente do Departamento de Terapia Ocupacional, Universidade Federal de São Carlos. E-mail: mirelafigueiredo@gmail.com 4.Docente do Departamento de Terapia Ocupacional da Universidade Federal de Minas Gerais. E-mail: cdrumonddebrito@gmail.com Endereço para correspondência: Departamento de Terapia Ocupacional da UFSCar. Rod. Washington Luís, Km 235 - SP-310. São Carlos, SP. CEP: 13565-905. 


\section{INTRODUÇÃO}

$\mathrm{O}$ câncer infanto-juvenil, que acomete crianças e adolescentes entre 0 a 18 anos, é considerado uma doença rara, que ocorre entre 1 a $3 \%$ na maioria das populações, sendo que em países em desenvolvimento o óbito por neoplasia ocorre em cerca de $1 \%$ dos casos. Os principais tipos de câncer infanto-juvenil são a leucemia, tumores do sistema nervoso central e linfomas ${ }^{1}$. As perspectivas de tratamento e cura são de $70 \%$, quando diagnosticadas precocemente. Tem-se a cirurgia, quimioterapia e radioterapia como tratamentos clínicos, sendo que alguns efeitos colaterais podem surgir de acordo com os diferentes tratamentos ${ }^{2}$.

A internação hospitalar na infância tem um impacto negativo, pois a criança é afastada da família, dos amigos e da escola, o que acontece quase sempre de modo repentino. Nessa situação, a criança perde suas referências afetivas e sociais, tendo que lidar com estresse, medo, insegurança e traumas, além de passar por tratamentos dolorosos e invasivos como a quimioterapia, radioterapia e cirurgias ${ }^{3}$.

Ainfância é um período de extrema importância para o desenvolvimento do ser humano em relação a aspectos físicos, motores, sociais, psicológicos e emocionais, e o crescimento humano depende da maturação biológica associada com a estimulação oferecida pelo meio ambiente desde os primeiros anos de vida ${ }^{4}$. Torna-se essencial que o tratamento de crianças e adolescentes com câncer ocorra por meio de uma equipe multiprofissional ancorada na interdisciplinaridade, sendo o Terapeuta Ocupacional um dos membros desta equipe ${ }^{5}$.

O Terapeuta Ocupacional é capacitado para analisar e avaliar o desenvolvimento infantil e tem papel fundamental em intervenções com crianças no contexto hospitalar ${ }^{6}$. Os objetivos destas intervenções com as crianças com câncer constituem em manter, estimular e reabilitar os componentes de desempenho ocupacional, sensório-motores, cognitivos e psicossociais prevenindo a incapacidade e promovendo a funcionalidade da criança. Suas intervenções também preconizam a utilização de estratégias para diminuição da dor, conservação de energia, prevenção de deficiências e deformidades e adaptação às mudanças trazidas pela doença nas atividades de vida diária básicas, instrumentais e de lazer evitando situações de incapacidade. Junto a criança, seus familiares e equipe multiprofissional o terapeuta ocupacional busca intervir auxiliando no enfrentamento da doença, na superação de possíveis complicações, na elaboração do luto vivido pelo paciente e pela família em virtude das diversas perdas inerentes à doença e ao tratamento, objetiva também resgatar a autoestima e o equilíbrio emocional do paciente, além de orientar e apoiar a família e cuidadores ${ }^{5}$.

As atividades lúdicas e criativas nesta fase do desenvolvimento são utilizadas nas intervenções terapêuticas ocupacionais, pois são recursos que promovem a continuidade do desenvolvimento global, da saúde mental da criança hospitalizada, a reorganização do cotidiano, a expressão da dor, da tristeza ou da desolação e favorece o vínculo entre paciente-terapeuta-família ${ }^{7}$. Esse profissional também indica dispositivos para acessibilidade como cadeira de rodas, andador, cadeira de banho e treina o uso dos mesmos. Também confecciona ou indica adaptações em objetos utilizados no dia a dia para compensar as limitações que podem ser causadas pelo tratamento ou pela própria doença $a^{8-9}$.

O Terapeuta Ocupacional, em conjunto com demais profissionais da equipe, atua com a família do paciente, pois a partir do diagnóstico da criança e do tratamento, a dinâmica familiar sofre uma desorganização em sua rotina, no seu cotidiano. Assim, como parte de ações inter e multiprofissionais no contexto da oncologia pediátrica, $\mathrm{o}$ terapeuta ocupacional pode propiciar orientações para que a família sinta mais segurança quanto aos procedimentos de tratamento; potencializar as redes de suporte e suas interlocuções; oferecer espaço de reflexão por meio do acompanhamento individual ou em grupos com mães e cuidadores; trocas e escuta qualificada; acolher as demandas; resgatar a presença de atividades significativas e quando necessário realizar o acolhimento da morte e acompanhamento pós-óbito 3 .

As discussões atuais sobre tratamento e cuidado em saúde que respondam para além da visão médicocentrada e cura da doença exigem intervenções pautadas pela integralidade. A consolidação do Sistema Único de Saúde (SUS) tem permitido a construção de novos paradigmas sobre o processo saúde-doença com repercussões na organização dos serviços de saúde, nas concepções de promoção à saúde, na prevenção de agravos e tratamento. Assim, a integralidade na atenção à saúde é um dos princípios finalísticos do SUS e orienta as políticas e ações programáticas que respondam às demandas e necessidades da população no acesso à rede de cuidados em saúde, considerando a complexidade e as especificidades de diferentes abordagens do processo saúde-doença e nas distintas dimensões, biológica, cultural e social do ser cuidado ${ }^{10}$. 
Nesse sentido, a Terapia Ocupacional busca responder às necessidades da vida atual complexa e singular, nos diferentes cursos de vida. Tem como propósito oferecer o cuidado integral ao indivíduo considerando contextos de vida e suas relações materiais e subjetivas. Atua como parte da equipe e busca responder ao conceito de integralidade defendido por $\operatorname{Mattos}^{11} \mathrm{o}$ qual se constrói na práxis do conjunto dos profissionais dos serviços de saúde e nas diferentes formas de encontro desses profissionais com e no serviço.

A presente pesquisa objetivou caracterizar o perfil do Terapeuta Ocupacional inserido no contexto do cuidado oncopediátrico. Esse perfil foi caracterizado tanto em relação as características demográficas e educacionais de cada profissional, portanto, a idade dos profissionais, o tempo de formação, entre outros aspectos, quanto em relação aos referenciais teóricos e/ ou abordagens que utilizam em suas práticas assistenciais com a família, com o paciente, e em sua inserção nas equipes multiprofissionais nas unidades de saúde em regime de internação.

\section{METODOLOGIA}

O presente estudo é descritivo de corte transversal ${ }^{12}$ e de abordagem quanti-qualitativa ${ }^{13}$

Foram estudados 11 terapeutas ocupacionais que atuam no contexto oncopediátrico no Estado de São Paulo. A identificação destes profissionais ocorreu mediante a listagem de instituições voltadas ao tratamento do câncer disponível pela Associação Brasileira de Linfomas e Leucemia (ABRALE), pelo Instituto Nacional do Câncer (INCA) e por instituições referenciadas por ambos, no final do ano 2012 e início de 2013.

Essa investigação adotou os seguintes critérios de inclusão: o terapeuta ocupacional deve trabalhar em instituições voltadas ao tratamento do câncer com a população infanto-juvenil; ter acesso online via internet para que fosse possível o envio do questionário; estar de acordo e assinar o Termo de Consentimento Livre e Esclarecido.

\section{Procedimentos e instrumento para coleta}

A coleta ocorreu mediante aprovação pelo Comitê de Ética em Pesquisa em Seres Humanos da Universidade Federal de São Carlos, sob o parecer n ${ }^{\circ}$ 231/2012.

Após a identificação e contato com os participantes, foi enviado por correio eletrônico um convite de participação no qual continha um link que direcionava ao instrumento da pesquisa. Este instrumento consistiu em um questionário online semi-estruturado e auto-aplicado elaborado pelas pesquisadoras juntamente com a Empresa Junior de Estatística da Universidade Federal de São Carlos.

O questionário era composto por 60 questões fechadas, prefixadas pelo pesquisador e que requeriam respostas afirmativas, negativas e/ou quantitativas referentes a dados sociodemográficos dos terapeutas ocupacionais (idade, sexo, ano de graduação, tipo de instituição de ensino, realização de pós-graduação, cargo na instituição); ao perfil de atuação (tipos de práticas realizadas, referenciais teóricos adotados, materiais e espaços utilizados, forma de atendimento individual ou grupal, com o paciente e com a família, atuação em equipe, média do número e duração dos atendimentos realizados semanalmente na instituição); caracterização dos pacientes atendidos por estes profissionais (idade, sexo, tipo de câncer). Além das questões fechadas, o questionário também continha duas questões abertas em que os entrevistados tinham a possibilidade de discorrer sobre as ações desenvolvidas conjuntamente com outros profissionais e descrever um atendimento que tivesse marcado sua carreira profissional.

As respostas das questões fechadas foram analisadas por meio de análise estatística simples de frequência e média sendo os resultados apresentados em forma de gráficos e quadros ${ }^{12}$. As respostas das questões abertas foram analisadas qualitativamente por meio da técnica de análise categorial destacando-se núcleos de sentido temático e cuja presença ou frequência de aparição propiciaram respostas para o objetivo analítico da pesquisa sendo apresentados os trechos das falas das participantes para ilustrar as vivências no campo de atuação ${ }^{13}$.

\section{RESULTADOS}

Foram encontrados na listagem de instituições voltadas ao tratamento do câncer 25 terapeutas ocupacionais na área oncopediátrica do Estado de São Paulo. Esses profissionais eram vinculados a 17 instituições das 101 identificadas inicialmente. Entretanto, do total de 25 profissionais listados somente 11 terapeutas ocupacionais, todas do sexo feminino, participaram da pesquisa respondendo o instrumento de coleta e compondo a amostra do presente estudo que teve as seguintes características descritas na Tabela 1. 
Tabela 1 - Características sócio demográficas dos participantes da pesquisa

\begin{tabular}{l|c|c|c|c|c|c}
\hline Faixa etária & $\mathbf{n}$ & Ano de graduação & $\mathbf{n}$ & $\begin{array}{c}\text { Instituição da } \\
\text { graduação }\end{array}$ & $\mathbf{n}$ & Pós-graduação \\
\hline & & 1980 & 2 & & & \\
& & 1998 & 1 & & & Doutorado \\
25 a 35 anos & 7 & 2000 & 1 & & Mestrado & 1 \\
35 a 45 anos & 2 & 2002 & 1 & Pública & 3 & 3 \\
45 a 55 anos & 2 & 2004 & 1 & & & Especialização \\
& & 2008 & 3 & & & Aprimoramento \\
& & 2011 & 1 & & & 8 \\
\hline
\end{tabular}

A atuação das terapeutas ocupacionais em termos panorâmicos foi delimitada pelos seus referenciais teóricos, métodos de atenção terapêutica ocupacional, tipos de serviços. Esses dados estão descritos nas Tabelas 2 e 3.

Destaca-se que uma das participantes trabalha nos três tipos de serviços citados.
Em relação ao perfil da população atendida, 7 participantes referem que atendem um número equivalente de crianças de ambos os sexos. As demais $(n=4)$ não souberam responder se há uma prevalência de gêneros em sua população atendida.

Tabela 2 - Local da pós-graduação latu sensu e referencial teórico utilizado

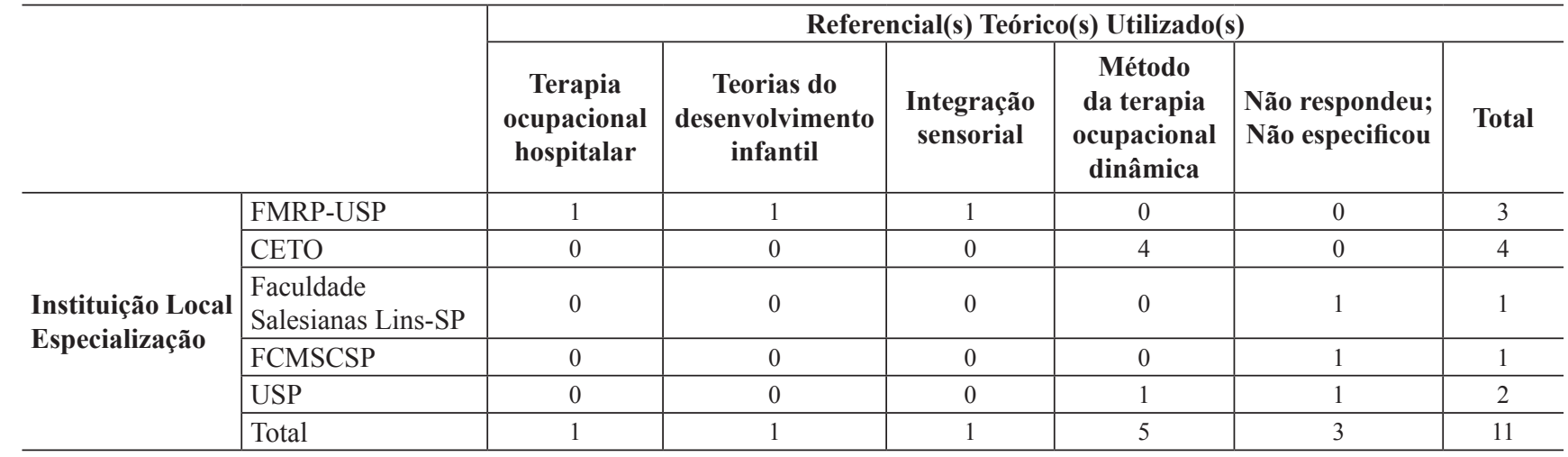

Tabela 3 - Nível de Atenção/ Tipos de serviços/ Referencial Teórico e abordagens terapêuticas

\begin{tabular}{l|l|l|l}
\hline Nível de atenção/tipos de serviço & $\mathbf{n}$ & Referencial Teórico e Abordagem terapêutica & $\mathbf{n}$ \\
\hline Serviço de nível terciário (geral, pré-cirúrgico, pós-cirúrgico) & 8 & $\begin{array}{l}\text { Método Terapia Ocupacional Dinâmica (MTOD) com } \\
\text { formação no Centro de Estudos em Terapia Ocupacional } \\
\text { (CETO)* }\end{array}$ & 4 \\
\hline Serviço de nível secundário (ambulatório, visita domiciliar) & 2 & Não especificou & 1 \\
\hline Casas de apoio & 3 & Terapia Ocupacional Hospitalar & 1 \\
\hline
\end{tabular}

Legenda: *O CETO e o MTOD foram fundados por Benetton sendo que o MTOD se constitui em um método de assistência em terapia ocupacional baseado em dois pressupostos fundamentais. O primeiro é que a prática seja sempre um processo de investigação e o segundo é que a proposição do uso das atividades como projeto terapêutico esteja claramente apresentada através de pressupostos teóricos e técnicos que fundamentem esse uso. Outras informações podem ser obtidas no site do CETO http://www.ceto.pro.br/atividades/index.php/inicio. 
A idade da população atendida esteve entre 1 a 15 anos, sendo que sete participantes relataram atender a população menor de 1 ano de idade; nove participantes atendiam a faixa etária de 1 a 4 anos; nove referiram atender os de 5 a 9 anos; nove responderam de 10 a 15 anos.

Os diagnósticos dos usuários dos serviços em que as participantes das pesquisas trabalhavam eram: leucemias $(81,82 \%)$, linfomas $(63,64 \%)$ e tumores do sistema nervoso central $(27,27 \%)$. Outros diagnósticos ainda mencionados: osteossarcoma, sarcoma de Ewing, retinoblastoma, neuroblastoma, câncer no fígado, rim, músculos, testículos e ovários, e transplantados de medula óssea.

Em relação ao tipo de atendimento, verificase que tanto o atendimento individual como o grupal são realizados com a clientela infanto-juvenil e seus respectivos familiares, como demonstrados no Gráfico 1 .

No que se refere a quantidade de atendimentos de pacientes e familiares realizados diariamente na instituição de trabalho dos terapeutas ocupacionais alvo da pesquisa, o número de atendimentos foram organizados em: de um a cinco atendimentos, entre cinco e dez, entre dez e 15 e mais de 15 atendimentos, como ilustrado no Gráfico 2 .
Nota-se que tanto para pacientes como para familiares, ocorre em maior frequência de 1 a 5 atendimentos de terapia ocupacional por dia. E o tempo médio de duração das sessões realizadas pelos terapeutas ocupacionais, tanto com o paciente quanto com os familiares, em sua maioria é de 30 minutos até 1 hora por sessão.

Em relação ao uso da atividade como recurso terapêutico, as participantes elencaram os principais materiais utilizados durante os atendimentos que foram classificados e agrupados em materiais gráficos, jogos, brinquedos, materiais artesanais, recursos midiáticos e eletrônicos.

Os materiais utilizados pela maioria das participantes foram os artesanais e os materiais gráficos ( $\mathrm{n}=5$ para cada um destes materiais). A utilização de brinquedos foi citada por 4 participantes e jogos, recursos midiáticos e eletrônico foram referidos por 3 participantes cada um. Ressalta-se que uma participante não respondeu a questão.

A maioria dos terapeutas ocupacionais $(n=8)$ referem que utilizam a brinquedoteca como um local de atendimento, 2 participantes não utilizam esse local e 1 não têm esse espaço como local de trabalho na instituição.

Gráfico 1 - Tipo de atendimento a pacientes e familiares

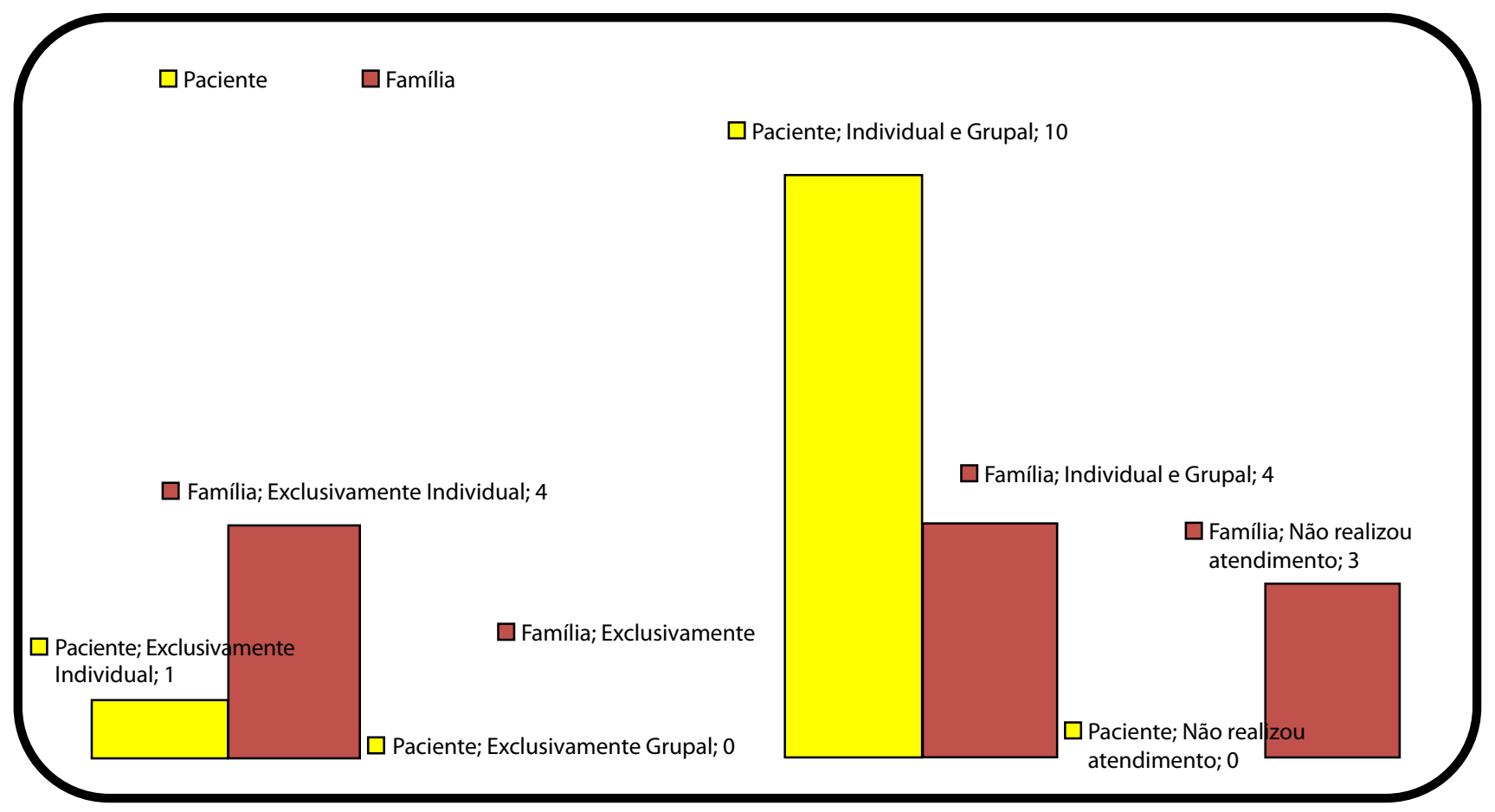


Gráfico 2 - Número de atendimentos de pacientes e familiares

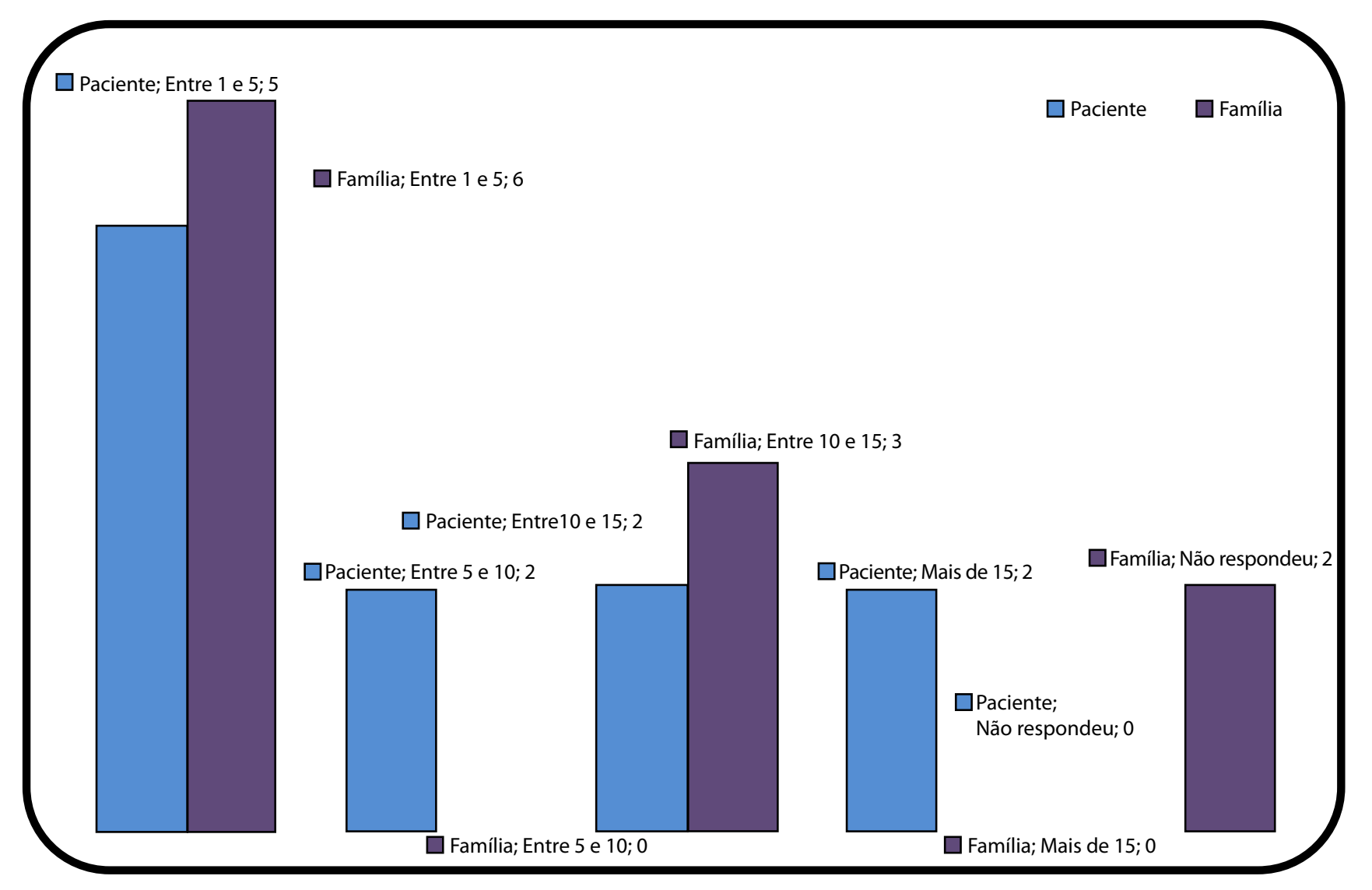

As participantes referem receber auxilio de outros membros da equipe multiprofissional durante os atendimentos, sendo que 1 recebe auxílio de um profissional, 5 recebem auxilio de dois profissionais e 3 recebem auxílio de mais de 2 profissionais. Importante destacar que 2 participantes atendem sozinhas. As participantes citam mais de uma categoria profissional que auxilia nos atendimentos do terapeuta ocupacional, sendo que os psicólogos foram os mais citados $(n=7)$, seguido pelos médicos $(n=6)$ e pela assistente social $(n=5)$. O fisioterapeuta e enfermeiro foram igualmente pontuados por 4 participantes cada. O fonoaudiólogo foi referido por 2 participantes e os profissionais nutricionista, farmacêutico e voluntários brinquedistas foram indicados por 1 participante cada um. Importante destacar que 2 participantes referiram que não recebem auxilio e 3 não responderam esta questão.
Em relação a quantidade de profissionais que auxiliam versus o quantitativo de pacientes atendidos, tem-se que a terapeuta ocupacional que recebia auxílio de um profissional atendia de 1 a 5 famílias de pacientes. As que possuíam ajuda de duas pessoas atendiam em sua maioria de 1 a 5 famílias, sendo que apenas uma atendia de 10 a 15 famílias. Já para as participantes que possuem mais de duas pessoas auxiliando, duas atendiam de 10 a 15 famílias e uma de 1 a 5 famílias.

Em relação às ações desenvolvidas conjuntamente com os outros profissionais, foram pontuadas as seguintes: reuniões de equipe (para estudo de casos clínicos e/ou para educação permanente); grupo de educação nutricional; orientações (de internação, de alta, à cuidadores e à familiares); atendimentos (multiprofissionais ${ }^{(1)}$ ), em conjunto, grupais, aos hóspedes, de intervenção precoce

(1) A atuação multiprofissional se configura como um trabalho coletivo organizado por profissionais de diversas categorias, cuja contribuição individual ressoa no produto final, atendendo às particularidades de cada área na solução de problemas diagnosticados em conjunto pelos seus membros ${ }^{14}$. 
junto com psicólogos, em cuidados paliativos e dor, para resolver uma questão específica ou interconsulta, isto é, a partir do encaminhamento de outro profissional da equipe; comemorações (festas de aniversário e temáticas); grupo do luto antecipatório; grupo de fila de espera; grupo de humanização; apoio ao óbito e atividades programadas com paciente(s) e seu(s) responsável(is). Observa-se que há uma variedade de categorias de ações realizadas com outros profissionais da equipe, sendo a reunião de equipe a mais pontuada pelas participantes $(n=5)$.

A reunião de equipe multiprofissional foi considerada importante como um instrumento para discutirem casos e auxilio para os atendimentos terapêuticos ocupacionais como exposto abaixo.

\footnotetext{
"A reunião semanal de equipe para discussão de todos os casos de pacientes que estão internados no momento [...] Considero que o auxílio se dá através das discussões de caso, para melhor compreensão do paciente e de seu familiar, o que auxilia indiretamente nos atendimentos de Terapia Ocupacional”. (Participante 1)
}

Sobre a descrição detalhada de um atendimento no qual as participantes tinham realizado e que havia marcado suas vidas ficou evidente a polarização da descrição do paciente baseada no modelo biomédico e outras descreviam aspectos biopsicossociais. Um modelo no qual valorizava a razão técnico-científica e priorização do diagnóstico e outro que valorizava aspectos subjetivos e do contexto. Duas narrativas exemplificam essa polarização:

"Adolescente, sexo masculino, diagnóstico de sarcoma, com metástase pulmonar e em diferentes ossos, internado por dor e desconforto respiratório". (Participante 10)

"[...] auto-imagem alterada, influenciando a auto-estima e a participação social, exige reestruturação na forma de realizar as atividades de vida diária e atividades de vida prática desde o início, seguidas das atividades ligadas à educação e ao lazer”. (Participante 1)

Os recursos terapêuticos utilizados, descritos em narrativas, tem materialidades diversas, alguns com materiais simples como papelão e velas. O impacto da intervenção terapêutica ocupacional é utilizar recursos dinâmicos, baseados no contexto e desejos dos pacientes, além de envolver a família. O lúdico e a diversão fazem parte da atenção terapêutica ocupacional.

\begin{abstract}
"Após cirurgias e sequelas devido ao tratamento ele falou que desejaria ser piloto de avião [...] Construímos então um avião de papelão e após bastante tempo e investimento de todos na construção enfim o voo foi marcado. No quintal da Casa de Apoio o voo foi de noite e acendemos estrelinhas (vela de aniversário) para caracterizar as turbinas. Foi uma experiência marcante, ele se divertiu muito, deu muitas risadas, voou com a mãe também”. (Participante 4)
\end{abstract}

A terapia ocupacional oncopediatrica trabalha diretamente com os familiares da criança e/ou adolescente, principalmente com as mães que são as cuidadoras na maior parte do tempo.

"Essa movimentação me marcou primeiro por refletir sobre a necessidade que essas mães e acompanhantes têm de cuidado e carinho tanto quanto seus filhos que estão doentes. E receber esse carinho dos seus filhos foi ainda mais significativo”. (Participante 9).

As participantes reconhecem o trabalho em equipe e têm postura proativa de outros profissionais.

“Assim, busquei o médico e a enfermeira responsáveis, discuti o caso e repassei detalhadamente todas as orientações para a paciente e sua mãe, destacando as possibilidades e não as restrições". (Participante 1)

A terapia ocupacional na oncologia exerce um papel importante na vida das crianças, adolescentes e familiares. Profissionais acolhem sonhos e desejos dos envolvidos no processo, busca autonomia e independência das crianças e adolescentes, exerce influência direta em suas qualidades de vida. Promove atendimento contextualizado e humanizado.

"Enfim na terapia ocupacional podemos concretizar sonhos, desejos e auxiliar na independência e autonomia do que o sujeito deseja para se tornar realidade. Tudo da forma como ele pode realizar e no tempo e limitação dele. A relação entre paciente, terapeuta e atividade é de fato o núcleo para a terapia ocupacional". (Participante, 4)

Desta forma, compreende-se que o trabalho do terapeuta ocupacional se constitui por uma relação triádica, composta por um terapeuta ocupacional, pelo indivíduo que apresenta qualquer tipo de motivo, necessidade e vontade de lá se encontrar para fazer terapia ocupacional e pelas atividades que possibilitam a ação terapêutica. 


\section{DISCUSSÃO}

A discussão proposta nesta pesquisa terá um cunho descritivo, pois a temática ainda é escassa na literatura científica brasileira e há necessidade de conhecer mais sobre a atuação do terapeuta ocupacional brasileiro com oncopediatria.

Os dados apresentados referentes às características sócio demográficas dos terapeutas ocupacionais (gênero, idade, tempo de formação e tipo de instituição de ensino) são similares aos encontrados no estudo realizado por Lima e Almohalha ${ }^{15}$, no qual buscou desvelar o papel de seis terapeutas ocupacionais na área de oncologia pediátrica em contextos hospitalares. Essa mesma semelhança se confirma nos estudos realizados por Sime et al. ${ }^{16}$ e GarciaSchinzari et al. ${ }^{8}$.

O presente estudo buscou ir além dos dados sócio demográficos e visou caracterizar as abordagens teóricas que apoiam a prática clínica dos terapeutas ocupacionais. Em relação às abordagens utilizadas, observa-se que dentre o escopo da amostra de 11 participantes, 27\% não especifica o modelo de suas intervenções e 36\% focam na terapia ocupacional dinâmica. Estudos de profissionais terapeutas ocupacionais dinâmicos ${ }^{17}$ atentam para que esses profissionais valorizam a experiência que as pessoas têm com a doença, tanto física, material quanto subjetiva e simbólica. Essa experiência faz sentido no processo de cuidar terapêutico ocupacional, pois é potencialmente capaz de ressignificar a vida, o cotidiano, a partir da experiência com a doença. Assim, é possível inferir que a escolha do método terapêutico ocupacional com pacientes oncológicos pode ter relação com processos humanos que ocorrem de aproximação com a temática da morte. Há uma aparente contradição que a morte nos convida, a de termos relações mais intensas com a vida. Os recursos terapêuticos ocupacionais têm como objetivo a construção da vida cotidiana a partir da repercussão gerada pela doença. O significado e desejos são elementos primordiais no raciocínio da terapia ocupacional que são acionados para desenvolver histórias capazes de transportar pessoas em condições complexas no processo saúde-doença para seu cotidiano a partir da relação triádica ${ }^{17}$, quer dizer, a partir da relação paciente, terapeuta e atividades.

Os materiais utilizados nas intervenções terapêuticas ocupacionais tiveram uma diversidade, destes elementos simples do cotidiano como papelão, cola, lápis, papel entre outros até termoplásticos de alta temperatura para confeccionar órteses, por exemplo. Os materiais são parte das diversas atividades propostas pelo terapeuta ocupacional a sua clientela. De acordo com Bartalotti e De $\mathrm{Carlo}^{18}$ as atividades permitem que o indivíduo seja reconhecido pelos seus afazeres, (re)descubra suas potencialidades e interesses, adquira novos conhecimentos, novas experiências de vida, transforme a rotina, modifique as interações sociais e possibilite a inclusão cultural. Desta forma, com a realização de atividades e utilização de inúmeros materiais, o terapeuta ocupacional pode (re) descobrir o potencial criativo do paciente, aumentar a sua autoestima, estimular a motivação e ajudar na manifestação dos sentimentos ${ }^{19}$.

Em consonância com Santos et al. ${ }^{6}$, os resultados deste estudo, demonstrou que a maioria dos terapeutas ocupacionais utiliza a brinquedoteca como um local de atendimento na atenção a crianças e adolescentes com diagnóstico de câncer. Segundo Santos et al. ${ }^{6}$, a brinquedoteca constitui como um recurso que pode ser utilizado, tanto pelos terapeutas ocupacionais como demais profissionais que atuam no contexto hospitalar para a humanização de hospitais. Esse espaço pode amenizar os efeitos do tratamento e ser utilizado como um setting terapêutico. Nesses ambientes o lúdico oferece suporte para atenuar a ruptura do cotidiano, da privação do brincar, do afastamento do núcleo familiar, de traumas, do medo e da ansiedade devido aos procedimentos médicos. Além disso, a brinquedoteca é um espaço para a interação entre crianças, no qual podem desempenhar o seu principal papel ocupacional que é o brincar. Santos et al. ${ }^{6}$ referem que apesar da existência de uma legislação (Lei 11.104 de 2005) que torna obrigatório à instalação de brinquedotecas nas unidades de saúde que ofereçam atendimento pediátrico em regime de internação, a presente pesquisa mostrou que um dos locais de trabalho não possuia brinquedoteca em suas dependências.

As respostas tornam evidente que a atuação do terapeuta ocupacional em oncopediatria envolve intervenções tanto com a criança como com a família. A literatura justifica esta intervenção definindo que o adoecimento e a hospitalização de uma criança ou adolescente afeta os pacientes e seus familiares e modifica a rotina de ambos. Assim, no trabalho realizado pelo terapeuta ocupacional se faz necessário a inclusão dos familiares ou a família estendida (avós, tios) e os amigos da criança, uma vez que todos podem auxiliar no processo do cuidado e de hospitalização $0^{20-21}$. Esta intervenção pode ocorrer por meio de uma escuta terapêutica, oferecendo informações ou estratégias de melhoria na qualidade de vida ${ }^{8,20}$.

A reunião de equipe, enquanto uma das ações desenvolvidas pelas terapeutas ocupacionais 
conjuntamente com os outros profissionais da equipe, também foi pontuada no estudo de Costa et al. ${ }^{22}$ que reafirmam a importância das reuniões de equipe enquanto um espaço para o diálogo, troca de ideias, experiências, discussão e reflexão acerca dos assuntos relativos à prática cotidiana dos profissionais na área da saúde, constituindo-se um meio de fortalecimento da equipe. Além disso, os autores referem que as reuniões não devem ser realizadas, apenas, para a cobrança do cumprimento de normas e rotinas da unidade ou para criticar o que está errado, mas devem servir para, a partir da identificação dos problemas, levantar sugestões, visando chegar a soluções mais concretas. Também, devem ser realizadas para elogiar, quando a equipe desempenha seu trabalho com qualidade. Desta forma, no setor de oncologia, o trabalho em equipe multiprofissional deve ser priorizado, uma vez que o paciente não é considerado como mais um caso, em uma abordagem holística e humanizada. Tais profissionais devem gerar não apenas saúde, mas principalmente vida $^{8,22}$.

Neste sentido, o terapeuta ocupacional na equipe, apresenta os resultados obtidos de sua relação com o usuário do serviço, na dinâmica das ocorrências dos três termos da relação triádica: paciente - terapeuta atividades ${ }^{23}$.

Em relação às descrições dos pacientes feitas pelas participantes deste estudo com base no modelo biomédico, o artigo de Mattingly ${ }^{23}$ corrobora apontando que parte dos profissionais tem realizado apresentações formais e biomédica de casos, focando a atenção na patologia e abordando os sintomas chaves, os principais acometimentos físicos típicos e as necessidades elementares. Entretanto, algumas participantes também descreveram os pacientes de forma a considerar os aspectos biopsicossociais, Mattingly ${ }^{23}$ também refere a existência de profissionais que apresentam os casos pautados no indivíduo e nas vivências que este enfrenta com a patologia e como esta o afeta em sua globalidade, ou seja em diferentes aspectos do sujeito.

\section{CONSIDERAÇÕES FINAIS}

O quantitativo de terapeutas ocupacionais deste estudo apresentou-se reduzido $(\mathrm{n}=11)$ apesar de existir 101 instituições que oferecem o tratamento oncológico no Estado de São Paulo. Com isso, destacam-se as limitações do estudo diante de uma amostra reduzida de participantes e a necessidade de continuar a produção científica nessa área, uma vez que após a revisão bibliográfica nos periódicos acadêmicos não foi encontrado diversidade de trabalhos científicos que abordassem o tema.

O perfil dos profissionais atuantes na área se caracterizou pelo sexo feminino, na faixa etária dos 25 aos 35 anos, conclusão da graduação em Terapia Ocupacional com maior incidência nos anos de 1980 e 2008, sendo a maioria com uma formação em instituição privada de ensino superior e que concluíram curso de pós-graduação lato-sensu no CETO utilizando como referencial teórico o MTOD.

A maioria das participantes realizavam atendimentos individuais e/ou grupais com os pacientes e/ou familiares, sendo de um a cinco atendimentos diários com duração média variando de 30 minutos a uma hora. Os terapeutas ocupacionais referiram receber auxílio no trabalho de outros profissionais, destacando-se os psicólogos e médicos. Consideram as reuniões de equipe multiprofissional importante como um instrumento para discussão de casos. Foram pontuados enquanto materiais utilizados nas atividades os jogos, brincadeiras lúdicas e o potencial que tais atividades podem representar na vida dos pacientes. $\mathrm{O}$ tipo de atuação descrita pelos terapeutas ocupacionais na oncopediatria envolveram aspectos como trabalho em equipe multiprofissional; discussão de caso; foco no sujeito e na família o que vai ao encontro de responder aos preceitos da política de saúde brasileira, em especial a da humanização visto ações de acolhimento, clínica ampliada e ambiência.

Cada participante relatou um atendimento que marcou sua vida de forma peculiar e fez descrições dos pacientes, sendo que parte apresentou uma narrativa voltada aos aspectos biopsicossociais dos pacientes e parte enfatizou a patologia e disfunções funcionais dos mesmos.

A partir dos relatos das participantes foi possível verificar ações realizadas pelo terapeuta ocupacional que atende às necessidades de intervenção com a clientela da área de oncopediatria, na medida em que são habilitados nas avaliações e elaboração de programas de intervenção que vão ao encontro das necessidades apresentadas pelas crianças e/ou adolescentes e seus respectivos familiares, assim como pela equipe de trabalho. Conclui-se assim, que a terapia ocupacional contribui para a integralidade do cuidado junto à gama de profissionais específicos necessários para o atendimento na área de oncopediatria. 


\section{REFERÊNCIAS}

1. Instituto Nacional de Câncer José Alencar Gomes da Silva (INCA). Estimativa 2014: incidência de câncer no Brasil. Rio de Janeiro: INCA; 2014. Disponível em: http://www. inca.gov.br/estimativa/2014/index.asp?ID=7.

2. Vasconcelos RF, Albuquerque VB, Costa ML G. Reflexões da clínica terapêutica ocupacional junto à criança com câncer na vigência da quimioterapia. Rev Bras Cancerol. 2006;52(2):129-37. Disponível em: http://www.inca.gov.br/ rbc/n_52/v02/pdf/artigo2.pdf.

3. Othero MB. Terapia ocupacional práticas em oncologia. São Paulo: Roca; 2010. p.3-15: Conceitos gerais em oncologia.

4. Bortolote GS, Bretas JRS. O ambiente estimulador ao desenvolvimento da criança hospitalizada. Rev Esc Enferm USP. 2008;42(3):422-9. doi: 10.1590/S008062342008000300002 .

5. Associação Brasileira de Linfomas e Leucemia (ABRALE). Terapia ocupacional na oncologia. In: Othero MB, organizador. Comitê de Terapia Ocupacional da Associação Brasileira de Linfoma e Leucemia; 2008. Mimeo.

6. Santos CA, Marques EM, Pfeifer LI. A brinquedoteca sob a visão da terapia ocupacional: diferentes contextos. Cad Ter Ocup UFSCar. 2006;14(2):91-102. Disponível em: http:// www.cadernosdeterapiaocupacional.ufscar.br/index.php/ cadernos/article/view/158/114.

7. Takatori M, Oshiro M, Otashima C. O hospital e a assistência em terapia ocupacional com a população infantil. In: De Carlo MMRP, Luzo MCM. Terapia ocupacional: reabilitação física e contextos hospitalares. São Paulo: Roca; 2004. p.256-77.

8. Garcia-Schinzari NR, Sposito AMP, Pfeifer LI. Cuidados paliativos junto a crianças e adolescentes hospitalizados com câncer: o papel da terapia ocupacional. Rev Bras Cancerol. 2013;59(2):239-47. Disponível em: http://www1. inca.gov.br/rbc/n_59/v02/pdf/11b-cuidados-paliativosjunto-a-criancas-e-adolescentes-hospitalizados-comcancer-o-papel-da-terapia-ocupacional.pdf.

9. Silveira AM, Joaquim RHVT, Cruz DMC. Tecnologia assistiva para promoção de atividades de vida diária com crianças em contexto hospitalar. Cad Ter Ocup UFSCar. 2012;20(2):183-90. doi: 10.4322/cto.2012.020.

10. Silva, KL, Sena RR. Integralidade do cuidado na saúde: indicações a partir da formação do enfermeiro. Rev Esc Enferm USP. 2008;42(1):48-56. doi: 10.1590/S008062342008000100007.
11. Mattos RA. A integralidade na prática (ou sobre a prática da integralidade). Cad Saúde Pública. 2004;20(5):1411-6. doi: 10.1590/S0102-311X2004000500037.

12. Goodwin JC. Research in psychology: methods and design. New York: John Wiley; 1995.

13. Turato ER. Tratado da metodologia da pesquisa clínicoqualitativa: construção teórico-epistemológica, discussão comparada e aplicação nas áreas da saúde e humanas. Petrópolis: Vozes; 2003.

14. Nunes MF, Wovst LR, Costa Neto SB. Trabalho em equipe: percepção interprofissional de uma clínica pediátrica. Rev Psicol Saúde. 2014;6(2):72-84. doi: 10.20435/pssa.v6i2.361.

15. Lima SL, Almohalha L. Desvelando o papel do terapeuta ocupacional na oncologia pediátrica em contextos hospitalares. Rev Ter Ocup Univ São Paulo. 2011;22(2):17281. Disponível em: http://www.revistas.usp.br/rto/article/ view/14135/92720.

16. Sime MM, Shishido NS, Santos WA. Caracterização do perfil da clientela do setor de terapia ocupacional na oncologia pediátrica. Rev Bras Cancerol. 2011;57(2):16775. Disponível em: http://wwwl.inca.gov.br/rbc/n_57/ v02/pdf/04_artigo_caracterizacao_perfil_clientela_setor_ terapia_ocupacional_oncologia_pediatrica.pdf.

17. Benetton J. A narrativa clinica no método terapia ocupacional dinâmica. Rev CETO. 2012;13(13). Disponível em: http:// www.ceto.pro.br/revistas/13/01-benneton.pdf.

18. Bartalotti CC, De Carlo MMRP, organizadores. Terapia ocupacional no Brasil: fundamentos e perspectivas. São Paulo: Plexus; 2001.

19. Othero MB, Palm RDCM. Terapia ocupacional em oncologia. In: Othero MB. Terapia ocupacional práticas em oncologia. São Paulo: Roca; 2010. p.72-122.

20. Palm RDCM. Oncologia. In: Cavalcanti A, Galvão C. Terapia ocupacional: fundamentação e prática. Rio de Janeiro: Guanabara Koogan; 2007. p.487-92.

21. Kudo AM, Pierri SAD. Terapia ocupacional com crianças hospitalizadas. In: Kudo AM, et al. Fisioterapia, fonoaudiologia e terapia ocupacional em pediatria. São Paulo: Sarvier; 1994. p.194-203.

22. Costa CA, Filho WDL, Soares NV. Assistência humanizada ao cliente oncológico: reflexões junto à equipe. Rev Bras Enferm. 2003;56(3):310-14. doi: 10.1590/S003471672003000300019 .

23. Mattingly C. A natureza narrativa do raciocínio clínico. Rev CETO. 2007;10(10):4-18. 